Marcus-Sebastian Martens Günter Thiel

\section{Ergebnisse und Prozesse im ambulanten Opiatentzug}

\author{
Outcome and Interactions in Outpatients Opiate Withdrawal
}

\section{Zusammenfassung}

$\mathrm{N}=111$ Heroinabhängige nahmen an einem ambulanten, methadongestützten und psychosozial begleiteten Entzugsprogramm teil. Eine Teilgruppe erhielt zusätzlich Akupunktur. Ergebnisse: Die EntzugsteilnehmerInnen waren relativ jünger und stärker sozial integriert mit bislang weniger Kontakt zum Drogenhilfesystem als die in der Basisdatendokumentation (BADO) der Hamburger Suchthilfe dokumentierte Klientel. Die Haltekraft nach zwei Wochen betrug $73 \%$. Nach mindestens zweiwöchiger Programmteilnahme erreichten $38 \%$ beim ersten und $46 \%$ beim zweiten Entzug das Entzugsziel mit negativer Abschlussurinkontrolle. KlientInnen mit fester Partnerbeziehung, zumindest Hauptschulabschluss, ohne Hepatitis C, mit weniger bisherigen Entzugsversuchen und einem späteren Beginn des Benzodiazepinkonsums schlossen häufiger den Entzug erfolgreich ab. KlientInnen mit höherer Heroindosierung vor dem Entzug hatten stärkeres „Drogenverlangen“, und sie brachen den Entzug häufiger ab. Eine schnellere Reduzierung des Drogenkonsums, weniger „Grübeln“ und weniger „Drogenverlangen“ standen im Zusammenhang mit der regulären Entzugsbeendigung. KlientInnen mit Akupunktur berichteten insgesamt nicht weniger Entzugsbeschwerden. Sie hatten auch keine besseren Entzugsergebnisse.

\section{Schliuisselwörter}

Ambulanter Entzug · methadongestützter Opiatentzug · Drogenabhängigkeit · Akupunktur

\section{Abstract}

$\mathrm{N}=111$ heroin dependent patients participated in an outpatient, methadone supported and psychosocially assisted withdrawal programme. A subgroup was additionally treated with acupuncture. Results: Participants of the withdrawal programme were younger and socially better integrated with less previous contacts to the drug help system than the clients documented in the Basic Data Documentation (BADO) of the Hamburg addiction help services. The retention rate after two weeks was $73 \%$. After at least two weeks' programme participation, $38 \%$ of the first-time withdrawal patients, and $46 \%$ of the second-time withdrawal patients were successful as shown by negative final urine controls. Clients living in stable partnerships, who had completed at least junior high school, who did not suffer from hepatitis C, had fewer withdrawal attempts and a later start of benzodiazepine use more often successfully concluded withdrawal treatment. Clients with higher heroin dosages prior to withdrawal treatment suffered more from craving and dropped out more frequently. A quicker reduction of drug use, less brooding and less craving were related with a regular conclusion of the withdrawal treatment. Clients receiving acupuncture did not, on the whole, report less withdrawal symptoms. They also did not have better withdrawal results.

\section{Key words}

Out patient opiate detoxification - methadone supported withdrawal $\cdot$ drug dependency $\cdot$ acupuncture 
Vor allem im englischsprachigen Raum weisen diverse Arbeiten seit längerem darauf hin, dass ambulante Opiatentzüge in verschiedenen Settings erfolgreich und sicher durchführbar sind [1 -9]. Im deutschsprachigen Raum wird der ambulante Opiatentzug seit einigen Jahren in verschiedenen Projekten praktiziert, und auch hier zeigen die veröffentlichten Ergebnisse, dass der ambulante Opiatentzug - tramadol-, codein-, methadonoder buprenorphingestützt - bei unterschiedlich zusammengesetzten Untersuchungsgruppen, unterschiedlichen Programmen und Erfolgskriterien von etwa einem Viertel bis zwei Drittel drogenfrei abgeschlossen wird [10-14].

In den Arbeiten von Bonorden-Kleij et al. [11], Piest [13] und Tsialtsudis et al. [10] gab es erste Indikationshinweise: KlientInnen, die zu Entzugsbeginn relativ besser sozial bzw. beruflich integriert waren oder in einer festen Partnerschaft lebten, beendeten den ambulanten Entzug häufiger erfolgreich. Sehr starker Heroinkonsum oder Benzodiazepinbeikonsum vor Entzugsbeginn reduzierten die Erfolgswahrscheinlichkeit beim ambulanten Entzug.

Zielsetzung der vorliegenden Studie war es zu untersuchen,

1. welcher Personenkreis durch ein ambulantes Entzugsprojekt erreicht wird und

2. wie die Entzugsverläufe und -ergebnisse sind.

3. Des Weiteren sollte überprüft werden, ob sich die angeführten Prädiktorvariablen auf Seiten der KlientInnen replizieren lassen und ob es weitere KlientInnenvariablen bzw. Prozessvariablen gibt, die im Zusammenhang mit erfolgreichen Entzugsabschlüssen stehen.

\section{Untersuchungsdurchführung}

In die Studie wurden alle KlientInnen einbezogen, die im Entzugsprojekt VIVA des Trägers Jugendhilfe e. V. in Hamburg innerhalb eines Jahres einen ambulanten Entzug begonnen hatten $(n=111)$. KlientInnen mit einem hohen Beigebrauch von anderen Suchtmitteln sowie mit erkennbaren schweren psychiatrischen und/oder stationär behandlungsbedürftigen körperlichen Erkrankungen wurden an entsprechende stationäre Entzugsstationen verwiesen.

Die Entzugseinrichtung war für 10 ambulante Entzugsplätze konzipiert und personell mit einer halben Arztstelle, zwei Krankenschwesterstellen sowie einer Sozialpädagogenstelle ausgestattet. Der Entzug wurde methadongestützt durchgeführt. Die KlientInnen erschienen täglich in der Entzugsambulanz, erhielten dort im Regelfall Methadon, ärztliche Betreuung und psychosoziale Begleitgespräche hinsichtlich der Entzugsbeschwerden und aktuellen Befindlichkeit. Die Anfangsdosierung beim methadongestützten Entzug betrug meist 4 Milliliter ( $M W=3.9$; $\mathrm{SD}=1.3$ ), maximal 10 Milliliter. Im Programm verpflichtend war die Teilnahme an einem Einzelgespräch pro Woche, zusätzlich bestand das Angebot einer wöchentlichen Gesprächsgruppe. Der direkte Betreuungsaufwand pro Klient und Woche lag bei 5 Stunden. Die nichtärztliche Betreuung war stark am Setting der klassischen Sozialarbeit orientiert. Die Gesprächsinhalte richteten sich nach den Bedürfnissen des Klienten.
Dem Entzugsprogramm lag kein spezifischer Ansatz für die psychosoziale Begleitung der Klienten zugrunde. Es kamen weder an Theorien orientierte, noch an psychotherapeutische Schulen angelehnte Interventionen und Begleitungen zum Einsatz.

Der Hälfte der KlientInnen wurde von Beginn der zweiten Jahreshälfte an ein begleitendes Akupunkturprogramm angeboten, an dem letztlich $\mathrm{n}=23$ teilnahmen. Die Akupunktur wurde als Ohrakupunktur nach dem NADA-Protokoll durch eine geschulte Krankenschwester im Mittel zweimal wöchentlich durchgeführt.

Die Klienten wurden während des Entzuges täglich vor der Methadonabgabe anhand einer einrichtungsintern entwickelten, 21 Items umfassenden Entzugssymptomliste zu ihrer physischen und psychischen Befindlichkeit und Schlafdauer befragt. Ausgewertet wurde die Anzahl an Tagen, an denen ein Symptom während des Entzugs auftrat, relativiert an der Entzugslänge in Tagen. Hierdurch wurde eine Wahrscheinlichkeit des Auftretens eines bestimmten Symptoms pro Tag ermittelt.

\section{Auswertung}

Retrospektiv extern ausgewertet und verrechnet wurden die in der einrichtungsinternen Dokumentation vorliegenden Anamnese- und Entzugsverlaufsdaten. An statistischen Prüfverfahren wurden je nach Datenqualität parametrische (t-Test, Varianzanalyse) bzw. nicht-parametrische Tests $\left(\chi^{2}\right.$-Test; Fisher-Test, Friedman-Test, Mann-Whitney-U-Test) und als Zusammenhangsmaße Rangkorrelationen nach Spearman bzw. Cramer-V verwendet. Es wurde jeweils mit zweiseitiger Fragestellung geprüft.

Ergebnisse

\section{Deskription der EntzugsteilnehmerInnen}

Die EntzugsteilnehmerInnen konnten hinsichtlich wesentlicher Anamnesemerkmale folgendermaßen näher beschrieben werden:

\section{Drogenkonsum}

Vor dem Entzug wurde bei $88 \%$ der Klienten täglicher Konsum von Heroin dokumentiert, bei $12 \%$ täglicher Konsum von Methadon, bei $16 \%$ Alkohol, bei $9 \%$ Kokain und bei 7\% Benzodiazepine. Clusteranalytisch (Brosius [15]) konnten unter Berücksichtigung der Häufigkeiten des Konsums von Heroin, Kokain, Benzodiazepinen, Methadon und Alkohol fünf Drogenkonsumtypen beschrieben werden: HeroinkonsumentInnen mit wenig weiterem Drogenkonsum (55\% der Gesamtgruppe); Heroin-/AlkoholkonsumentInnen (14\%), Heroin-/KokainkonsumentInnen (12\%), Methadonsubstituierte mit häufigerem Heroin-, Alkoholkonsum (11\%) und HeroinkonsumentInnen mit Benzodiazepinkonsum (7\%). Im Mittel gaben die EntzugsteilnehmerInnen 6,7 Jahre Heroinkonsum an.

\section{Soziodemographische Merkmale}

79\% der Entzugsteilnehmer waren Männer mit einem Altersmittelwert von 29,8 Jahren, $21 \%$ waren Frauen mit einem Altersmittelwert von 27,6 Jahren; $22 \%$ befanden sich in der Alterskategorie bis 21 Jahre. $85 \%$ der EntzugsteilnehmerInnen waren deutscher Nationalität. 21 \% hatten keinen Hauptschulabschluss, 
44\% Hauptschulabschluss, $24 \%$ mittlere Reife, $11 \%$ Hochschulreife. $45 \%$ konnten eine abgeschlossene Berufsausbildung aufweisen. $50 \%$ hatten Vorstrafen, $38 \%$ Hafterfahrungen.

\section{Soziale Situation}

$38 \%$ der Entzugsteilnehmer standen in einem Arbeitsverhältnis. Ihren Lebensunterhalt finanzierten 36\% über Sozialhilfe. 70\% waren verschuldet. 58\% lebten in einer eigenen Wohnung allein oder in Partnerschaft, 29\% bei Angehörigen. 75\% waren ledig, 9\% verheiratet. Jede/r Vierte (28\%) hatte Kinder. 59\% lebten in fester Partnerschaft.

\section{Kontakt zum Drogenhilfesystem}

$55 \%$ hatten bisher keinen regelmäßigen Kontakt zu einer Drogenhilfeeinrichtung. $55 \%$ hatten bereits mindestens einen stationären Entzug begonnen, 26\% mindestens eine stationäre Therapie und $8 \%$ mindestens eine ambulante Therapie. $31 \%$ waren bereits substituiert gewesen.

\section{Gesundheitszustand}

Gemäß ärztlicher Eingangsuntersuchung wiesen 70\% einen unauffälligen körperlichen Allgemeinzustand auf, bei 57\% war der Zahnstatus unauffällig. 38\% kamen mit Hepatitis C, $17 \%$ mit Hepatitis B, $2 \%$ mit HIV-Infektion, $3 \%$ mit Abszessen. $28 \%$ berichteten von Suizidversuchen im Laufe ihres Lebens.

\section{Entzugsverlauf}

Unter Berücksichtigung aller ProgrammteilnehmerInnen dauerte der erste Entzug im Mittel drei Wochen $(M W=21$ Tage; $S D=11$ ). Ein regulärer Abschluss des Entzugs lag vor, wenn der Klient kein Substitut mehr erhielt und die letzte Urinkontrolle negativ war. Dieses Entzugsziel erreichten 27\% aller in das Programm eingetretenen Klienten nach dem ersten Entzug.

35 Klienten (31\%) unterzogen sich einem zweiten Entzug. Der zweite Entzug begann im Mittel 2,5 Monate nach dem ersten Entzug. 34\% der Klienten konnten diesen regulär abschließen. Der zweite Entzug dauerte im Durchschnitt ebenfalls etwa drei Wochen (MW = 22 Tage). Zusammengefasst: Von 111 KlientInnen, die einen Entzug begonnen hatten, konnten 31\% einmal den Entzug regulär mit negativer Urinkontrolle und ohne Substitutionsmittel abschließen.

Im Mittel dauerten die regulär abgeschlossenen Entzüge 27 Tage $(S D=10)$ und die abgebrochenen Entzüge 19 Tage $(S D=10$; $\mathrm{p}<0,001)$. Die regulär beendeten zweiten Entzüge dauerten im Mittel 29 Tage $(S D=10)$ und die abgebrochenen 18 Tage $(S D=10 ; p<0,001)$. KlientInnen mit regulär abgeschlossenem ersten Entzug beendeten den zweiten Entzug häufiger regulär als KlientInnen mit abgebrochenem ersten Entzug $(p<0,05)$.

Bis zum Ende der zweiten Woche befanden sich noch $73 \%$, bis zum Ende der dritten Woche noch 53\% aller aufgenommenen KlientInnen im Entzugsprogramm. 38\% derjenigen mit mindestens 14-tägiger Teilnahme am Entzugsprogramm erreichten letztlich das Entzugsziel völliger Drogenfreiheit. Beim zweiten Entzug lag dieser Anteil bei 46\%. Diejenigen mit mindestens dreiwöchiger Entzugsteilnahme schafften beim ersten Entzug zu $51 \%$ und beim zweiten Entzug zu 86\% das Entzugsziel.
Betrachtet man den ersten Entzugsprozess bei allen KlientInnen unter Berücksichtigung der Urinkontrollergebnisse, so zeigte sich bis zum Ende der dritten Entzugswoche der Entzugsfortschritt: In der Eingangsurinkontrolle wurden im Mittel 2,1 verschiedene Substanzen festgestellt. Nach einer Woche sank der Mittelwert auf 1,2, nach zwei Wochen auf im Mittel 0,9 und nach drei Entzugswochen auf 0,6 verschiedene Drogen $(p<0,0001)$.

\section{Prädiktion des Entzugsergebnisses}

Es wurde überprüft, welche der zu Entzugsbeginn erhobenen KlientInnenmerkmale bzw. welche Behandlungs- oder Prozessmerkmale im Zusammenhang mit dem Entzugsergebnis (regulärer Abschluss mit negativer Urinkontrolle vs. Entzugsabbruch) standen.

\section{KlientInnenmerkmale}

Der Entzug wurde öfter regulär abgeschlossen, wenn die KlientInnen in einer festen PartnerIn-Beziehung lebten, relativ später mit dem Benzodiazepinkonsum begonnen hatten, relativ weniger Entzüge - privat oder institutionell - versucht hatten, zumindest einen Hauptschulabschluss besaßen, von der Entzugseinrichtung relativ weiter entfernt wohnten und keine Hepatitis $C$ hatten. Alle Einzelzusammenhänge waren nicht größer als $R=0,29$ (jeweils $\mathrm{p}<0,05)$. Tendenziell beendeten auch KlientInnen mit Kindern etwas häufiger den Entzug $(p<0,10)$. Alle weiteren KlientInnenvariablen standen in keinem Zusammenhang mit dem Entzugsergebnis.

Die verschiedenen Drogenkonsumtypen erzielten keine unterschiedlichen Entzugsergebnisse, jedoch schlossen KlientInnen mit höherer Heroindosierung seltener den Entzug ab (Abb.1).

Während der erste Entzug von den beiden Teilgruppen mit niedrigerer Heroindosierung von $32 \%$ bzw. 39\% regulär abgeschlossen wurde, lag dieser Anteil bei der am stärksten dosierten Teilgruppe $(n=35)$ bei $9 \%(p<0,01)$.

\section{Behandlungs- und Prozessvariablen}

KlientInnen, bei denen am Ende der zweiten Entzugswoche relativ mehr Drogen festgestellt wurden, beendeten seltener den Entzug regulär $(\mathrm{p}<0,05)$.

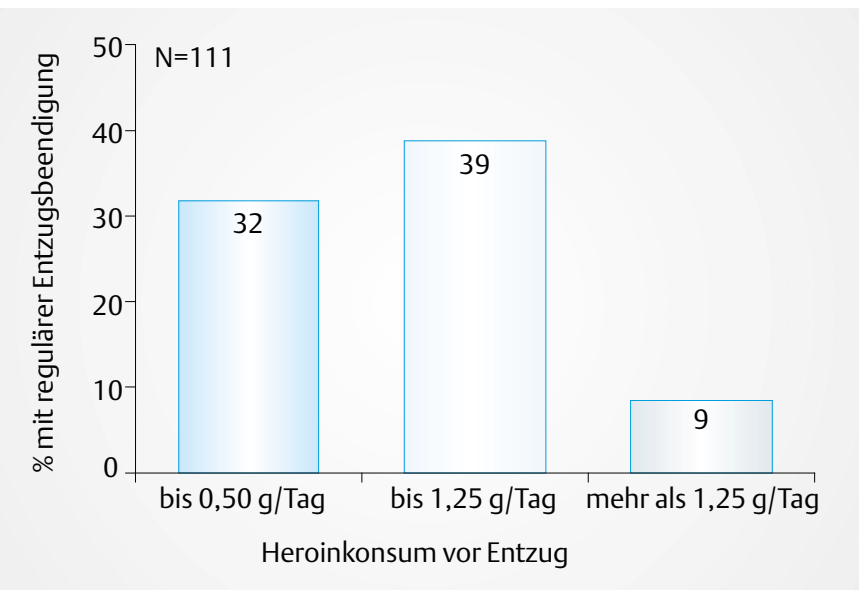

Abb. 1 Heroindosierung vor dem Entzug und Entzugserfolg. 
Etwas häufigere psychische Entzugsbeschwerden wie „Grübeln“ $(p<0,05)$, „innere Unruhe“ $(p<0,10)$ sowie vor allem „Drogenverlangen“ ( $p<0,0001)$ standen im Zusammenhang mit dem Entzugsabbruch. Körperliche Entzugsbeschwerden traten bei Entzugsabbrechern und Entzugsabschließern gleich häufig auf (Abb. 2). KlientInnen mit höherer Heroindosierung (mehr als $1,25 \mathrm{~g} / \mathrm{Tag}$ ) hatten die gleichen Entzugsbeschwerden wie weniger stark dosierte. Ausnahme: Sie hatten deutlich häufigeres „Drogenverlangen“ ( $p<0,01)$.

Die Schlafdauer der regulären EntzugsbeenderInnen war mit 7 Stunden pro Nacht genauso lang wie bei den Entzugsabbrechern. Im Mittel beklagten sich die EntzugsteilnehmerInnen nur jeden dritten bis vierten Tag über Schlaflosigkeit.

\section{Akupunktur}

Die TeilnehmerInnen am zusätzlichen Akupunkturprogramm $(\mathrm{n}=23)$ unterschieden sich von den übrigen EntzugsteilnehmerInnen nur in einem von 23 Anamnesemerkmalen (u.a. Alter, Geschlecht, Nationalität, Schul- und Berufsbildung, Wohnsituation, Partnerbeziehung, Behandlungserfahrungen, Konsumverhalten, Gesundheitszustand), und zwar gaben sie häufiger sporadischen Kokainkonsum an $(\mathrm{p}<0,01)$.
Die AkupunkturteilnehmerInnen berichteten insgesamt nicht über weniger Entzugsbeschwerden (Abb. 3), und sie beendeten den Entzug auch nicht häufiger regulär. Allerdings beendeten KlientInnen mit relativ geringerem Heroinkonsum vor Entzugsbeginn - bis zu 0,50 g/Tag - tendenziell häufiger den Entzug erfolgreich, wenn sie am Akupunkturprogramm teilgenommen hatten $(\mathrm{p}<0,10)$.

\section{Diskussion der Ergebnisse}

Im ambulanten Entzug wurden Drogenabhängige erreicht, die verglichen mit den umfangreichen Daten der Hamburger Basisdatendokumentation (Simmedinger et al. [16]) - eine um etwa sechs Jahre kürzere Drogenkarriere mit deutlich weniger stationären Entzugs- und Therapieerfahrungen hinter sich haben und im Durchschnitt etwa drei Jahre jünger waren. Vor allem wurden in der Altersgruppe bis 21 Jahre relativ mehr erreicht. Deutlich weniger waren vorbestraft. Die Entzugsteilnehmer standen häufiger in einem Arbeitsverhältnis. Sie bezogen seltener Sozialhilfe. Sehr viel mehr lebten in einer festen Partnerschaft. In der ärztlichen Aufnahmeuntersuchung wurde offensichtlich insgesamt ein relativ besserer Gesundheitszustand festgestellt, als Brandt et al. [17] ihn für zwei Hamburger stationäre Entzugseinrichtungen berichten. Hingewiesen werden
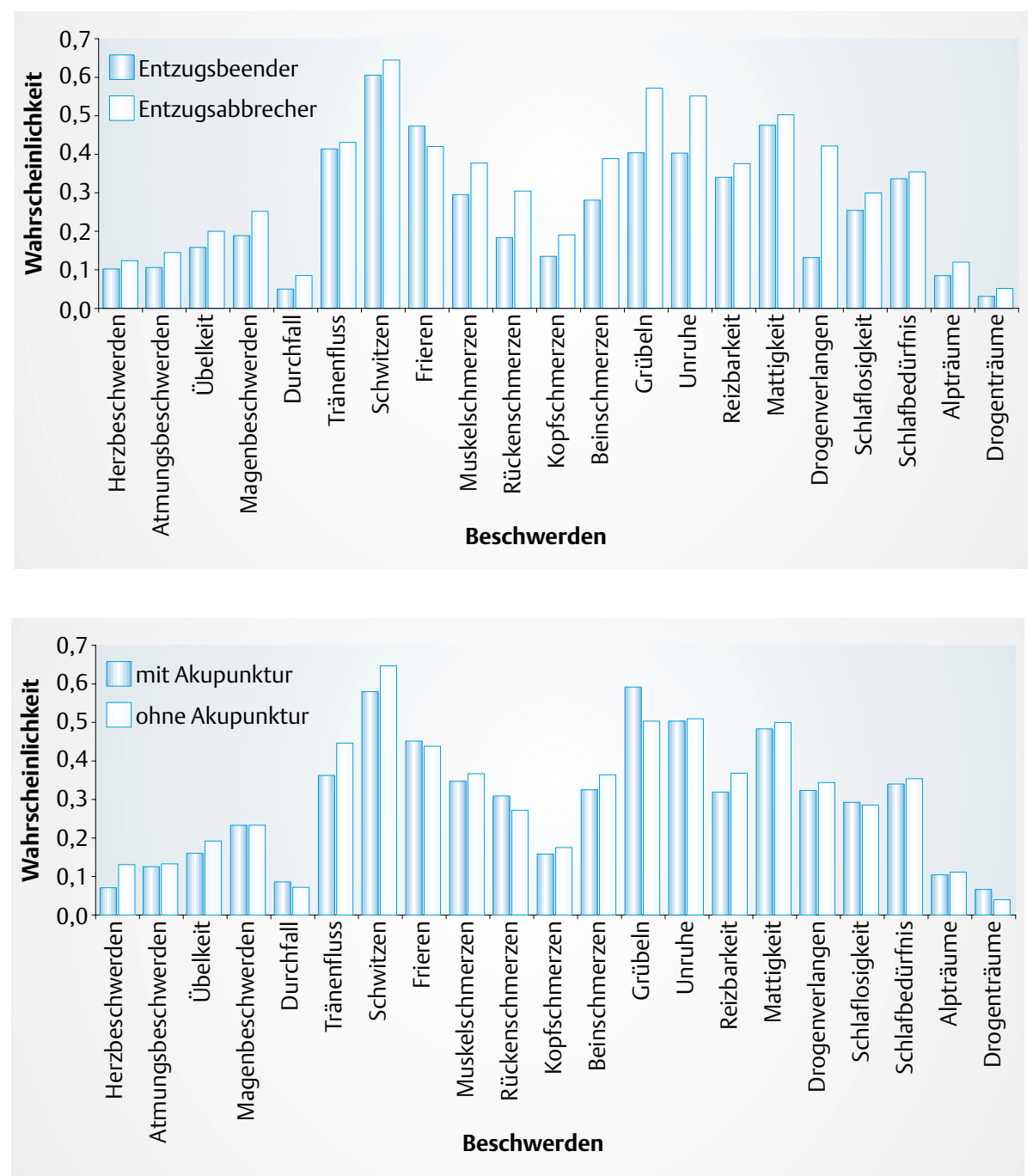

Abb. 2 Entzugsbeschwerden von regulären Entzugsbeendern und -abbrechern.

Abb. 3 Akupunktur und Entzugsbeschwerden. 
soll auch auf den Befund, dass etwa jede/r Zweite keinen regelmäßigen Kontakt zum Drogenhilfesystem hatte. Beim Vergleich mit der Klientel der Hamburger stationären Entzugseinrichtungen (Brandt et al. [17]) zeigt sich, dass die KlientInnen im ambulanten Entzug seltener vorbestraft waren, häufiger in fester Partnerschaft lebten, seltener substituiert waren und seltener Kontakt zum Drogenhilfesystem hatten.

Insgesamt kann resümiert werden, dass durch den ambulanten Entzug ein vergleichsweise jüngerer und noch stärker sozial integrierter Personenkreis mit weniger Gesundheitsproblemen in einem relativ frühen Suchtstadium erreicht wurde. Über den ambulanten Entzug eröffnen sich somit Möglichkeiten, Drogenabhängige erstmals an Hilfsangebote heranzuführen, gesundheitspräventive Botschaften $\mathrm{zu}$ übermitteln, suchttypische Erkrankungen rechtzeitiger zu behandeln und lange Suchtkarrieren zu verkürzen. Bereits Tsialtsudis et al. [10] zeigten auf, dass über das ambulante Entzugsangebot sehr viele KlientInnen ohne bisherigen Kontakt zum Drogenhilfesystem erreicht wurden.

Die Haltequote war mit 73\% nach 14 Tagen sehr hoch, und von denjenigen mit mindestens zweiwöchiger Programmteilnahme erreichte beim ersten Entzug gut jede/r Dritte (38\%), beim zweiten Entzug fast jede/r Zweite (46\%) das Entzugsziel. Bei einer mindestens dreiwöchigen Entzugsteilnahme lagen die Anteile erfolgreich Entgifteter beim ersten Entzug bei 51\% und beim zweiten Entzug bei 86\%. Die Ergebnisse bewegen sich somit in der bisher bekannten Größenordnung, wobei die durchschnittliche Entzugsdauer gegenüber der ersten Studie im Projekt VIVA (Bonorden-Kleij [11]) deutlich reduziert wurde. Die Ergebnisse unterstreichen, dass bei einer hohen Haltequote der ambulante Opiatentzug möglich ist. Die Entzugsverläufe zeigten, dass eher medizinisch unproblematische Entzugssymptome wie Schwitzen, Frieren, Muskelschmerzen und verstärkter Tränenfluss dominant sind, also Symptome, die in einem ambulanten Setting gut zu beherrschen sind.

Die Annahme, dass KlientInnen mit fester Partnerschaft den ambulanten Entzug häufiger erfolgreich abschließen (BonordenKleij [11]), konnte in dieser Studie bestätigt werden. Nicht jedoch ergab sich wie bei Bonorden-Kleij [11] sowie Piest [13], dass Erwerbstätige häufiger den Entzug erfolgreich abschlossen.

Während bei Piest [13] Benzodiazepinkonsum vor Entzugsbeginn ein negativer Prädiktor war, stand in dieser Arbeit ein früher Einstieg in den Benzodiazepinkonsum im Zusammenhang mit dem Entzugsabbruch. Gleichermaßen beendeten KlientInnen mit hoher Heroindosierung seltener den Entzug, obwohl diese mit Ausnahme von „Drogenverlangen“ keine anderen Entzugsbeschwerden berichteten als niedriger Dosierte.

Der Befund von Tsialtsudis et al. [10], dass KlientInnen mit starkem Heroinkonsum schlechtere Ergebnisse im ambulanten Entzug haben, konnte in dieser Studie repliziert werden.

Der Entzug wurde häufiger abgeschlossen, wenn KlientInnen Kinder hatten, bisher weniger Entzüge versucht bzw. zumindest einen Hauptschulabschluss besaßen bzw. aus von der Entzugseinrichtung entfernteren Stadtteilen kamen. Vermutlich stellte der letztere Personenkreis eine stärkere Auslese hinsichtlich der
Entzugsmotivation dar, da größere Anstrengungen zur Teilnahme am Entzugsprogramm unternommen werden mussten. Schließlich schlossen auch KlientInnen mit weniger Gesundheitsproblemen (keine Hepatitis C) den Entzug häufiger ab. Alle bivariaten Zusammenhänge waren schwach. Inwieweit sich diese KlientInnen-Merkmale als bedeutsam für den Entzugserfolg replizieren lassen, bedarf weiterer Studien.

Wie in der Arbeit von Schmidt et al. [14] deutete sich hier an, dass Entzugsabbrecher während des Entzugs ein deutlich stärkeres Drogenverlangen hatten. Zusätzlich wurden in der vorliegenden Untersuchung „innere Unruhe“ und „Grübeln“ als schwache Prädiktorvariablen für häufigere Entzugsabbrüche festgestellt. In der begleitenden psychosozialen Betreuung erlangt offensichtlich die Hilfe zur Bewältigung dieser psychischen Entzugsbeschwerden hohe Bedeutung. Es sollte in folgenden empirischen Untersuchungen der Hypothese nachgegangen werden, ob eine stärker standardisierte, theoriegeleitete und zeitlich intensivere psychosoziale Begleitung die psychischen Entzugssymptome deutlich reduzieren kann und den relativen Anteil an regulären Beendern signifikant erhöht.

Die nach 14 Entzugstagen festgestellte unterschiedliche Anzahl von Drogen bei Abbrechern und regulären Beendern lässt vermuten, dass spätere reguläre EntzugsbeenderInnen offensichtlich schon zu einem früheren Zeitpunkt zielstrebiger entgiften.

Während es neben positiven klinischen Erfahrungen (vgl. Strauß et al. [18], [19]) einige Studien mit Hinweisen auf die Wirksamkeit von Akupunktur beim Opiatentzug gibt (z. B. Brewington et al. [20]; Shwartz et al. [21]), konnte in dieser Arbeit ähnlich wie bei Piest [13] keine Auswirkung auf Entzugsbeschwerden und Entzugsverlauf festgestellt werden. Auch Verthein et al. [22] stellten fest, dass die Akupunktur keinen Einfluss auf eine Verringerung des Heroinkonsums hatte und offensichtlich nicht substanzunspezifisch wirkt. Es ließ sich eher eine gewisse Wirksamkeit bei Kokain- oder Alkoholabhängigen erkennen (Avants et al. [23]). Es bedarf weiterer Überprüfungen, ob zum Erreichen nachweisbarer Effekte z.B. eine tägliche Akupunkturfrequenz erforderlich ist (Strauß et al. [19], Stiller et al. [24]). Margolin et al. [25] konnten zeigen, dass Akupunktur als „stand-alone“-Behandlung für Kokainabhängige nicht ausreichend ist, und forderten die Überprüfung von Akupunktur als unterstützende Behandlungsmaßnahme. Die in der vorliegenden Studie aufgezeigte Tendenz, dass KlientInnen mit Akupunkturunterstützung häufiger den Entzug erfolgreich abschlossen, wenn sie vor Entzugsbeginn nur geringeren Heroinkonsum hatten, deutet die Komplexität möglicher Wirkungszusammenhänge an.

\section{Literatur}

${ }^{1}$ DelCampo EJ, John DS, Kauffman CC. Evaluation of the 21-day outpatient heroin detoxification. Int J Addict 1977; 12 (7): 923 - 935

2 Sorensen JL, Hargreaves WA, Weinberg JA. Withdrawal from heroin in three or six weeks. Comparison of methadyl acetate and methadone. Arch Gen Psychiatry 1982; 39 (2): 167-171

${ }^{3}$ Rounsaville BJ, Kosten T, Kleber H. Success and failure at outpatient opioid detoxification. Evaluating the process of clonidine and methadone assisted withdrawal. J Nerv Ment Dis 1985; 173 (2): 103 - 110 
${ }^{4}$ Gossop M, Strang J. A comparison of the withdrawal responses of heroin and methadone addicts during detoxification. Br J Psychiatry 1991; 158: $697-699$

${ }^{5}$ Kleber HD, Topazian M, Gaspari J et al. Clonidine and naltrexone in the outpatient treatment of heroin withdrawal. Am J Drug Alcohol Abuse 1987; $13(1-2): 1-17$

${ }^{6}$ O'Connor PG, Waugh ME, Carroll KM et al. Primary care based ambulatory opioid detoxification: the results of a clinical trial. J Gen Intern Med 1995; 10 (5): 255-260

${ }^{7}$ McCann MJ, Miotto K, Rawson RA et al. Outpatient non opioid detoxification for opioid withdrawal. Who is likely to benefit? Am J Addict 1997; 6 (3): $218-223$

${ }^{8}$ Bickel WK, Amass L, Higgins ST et al. Effects of adding behavioral treatment to opioid detoxification with buprenorphine. J Consult Clin Psychol 1997; 65 (5): $803-810$

${ }^{9}$ Gruber K, Chutuape MA, Stitzer ML. Reinforcement-based intensive outpatient treatment for inner city opiate abusers: a short-term evaluation. Drug Alcohol Depend 2000; 57 (3): 211 - 223

${ }^{10}$ Tsialtsudis B, Chorzelski G, Krausz M. Ambulanter Drogenentzug mit Tramadol. In: Behrendt K, Degkwitz P, Trüg E (Hrsg). Schnittstelle Drogenentzug. Freiburg im Breisgau: Lambertus-Verlag 1995; 167- 177

${ }_{11}$ Bonorden-Kleij K, Puttkammer S. Ambulanter Entzug. In: Behrendt K, Degkwitz P, Trüg E (Hrsg). Schnittstelle Drogenentzug. Freiburg im Breisgau: Lambertus-Verlag 1995; $154-166$

${ }^{12}$ Diamant K, Fischer G, Schneider C et al. Outpatient opiate detoxification treatment with buprenorphine. Preliminary investigation. Eur Addict Res 1998; 4 (4): 198 -202

13 Piest B. Ambulanter Entzug von Opiaten. Sucht 1999; 45 (4): 263 -268

${ }^{14}$ Schmidt M, Krahl W, Holst A et al. Tagesklinische Entzugsbehandlung Opiatabhängiger. Sucht 1999; 45 (3): 195-200
${ }^{15}$ Brosius F. SPSS 8 Professionelle Statistik unter Windows. Bonn: MITPVerlag 1998

${ }^{16}$ Simmedinger R, Schmid M, Vogt I. Ambulante Suchthilfe in Hamburg. Statusbericht 2000 zur Hamburger Basisdatendokumentation im ambulanten Suchthilfesystem. ISS Frankfurt am Main 2001

${ }^{17}$ Brandt J, Behrendt K, Wyszomirski D et al. Zur qualifizierten Entgiftung Hamburger Drogenabhängiger. Sucht 1999; 45 (2): 117-119

${ }^{18}$ Strauß K, Weidig W. Akupunktur im Drogenentzug - ein kurzer Abriß. In: Behrendt K, Degkwitz P, Trüg E (Hrsg). Schnittstelle Drogenentzug. Freiburg im Breisgau: Lambertus-Verlag 1995; $122-140$

${ }^{19}$ Strauß K, Weidig W (Hrsg). Akupunktur in der Suchtmedizin. Stuttgart: Hippokrates Verlag 1997

20 Brewington V, Smith M, Lipton D. Acupuncture as a detoxification treatment: An analysis of controlled research. Journal of Substance Abuse Treatment 1994; 11 (4): 289-307

${ }^{21}$ Shwartz M, Saitz R, Mulvey K et al. The value of acupuncture detoxification programs in a substance abuse treatment system. Journal of Substance Abuse Treatment 1999; 17 (4): 305-312

${ }^{22}$ Verthein U, Raben R, van Soer J. Ambulante Akupunkturbehandlung bei Drogen- und Alkoholabhängigen - Ergebnisse einer Verlaufsuntersuchung. Sucht 2000; 46 (1): $62-76$

${ }^{23}$ Avants SK, Margolin A, Holford TR et al. A randomized controlled trial of auricular acupuncture for cocaine dependence. Arch Intern Med 2000; 160 (15): 2305-2312

${ }^{24}$ Stiller N, Stux G, Pomeranz B. Akupunktur - Lehrbuch und Atlas. 5.Auflage. Berlin: Springer-Verlag 1999

${ }^{25}$ Margolin A, Kleber HD, Avants SK et al. Acupuncture for the treatment of cocaine addiction: a randomized controlled trial. JAMA 2002; 287 (1): $55-63$

\section{Suchttherapietage in Hamburg 10.-13. Juni 2003}

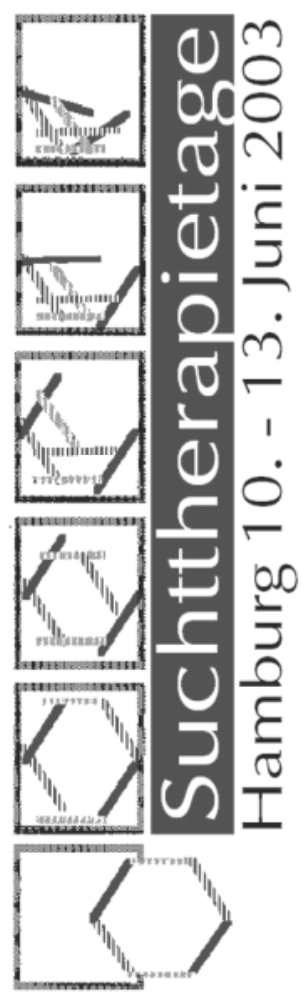

Schwerpunktthema: Sucht ohne Suchtmittel. Exzessives Verhalten, pathologisches Spielen, Sexsucht, Internetsucht und Essstörungen

Im Tagungsmittelpunkt steht das Thema „Sucht ohne Suchtmittel. Exzessives Verhalten, pathologisches Spielen, Sexsucht, Internetsucht und Essstörungen - psychische Krankheit oder Modeerscheinung?". Damit widmen sich die Suchttherapietage einem Themengebiet, das in seiner Bedeutung für den Lebensalltag der Betroffenen einerseits sowie der therapeutischen Arbeit andererseits den klassischen Süchten jedoch nicht nachsteht. Verschiedene exzessive Verhaltensweisen werden in der Öffentlichkeit und in professionellen Kreisen zunehmend in die Nähe von Sucht gerückt und als neues Phänomen diskutiert. Zur Thematik „Sucht ohne Suchtmittel“ werden grundsätzliche Fragen der Vergleichbarkeit der Verhaltensweisen ebenso erörtert wie Fragen der Diagnostik und geeigneter Interventionsstrategien.

In über 100 Einzelveranstaltungen zum Schwerpunktthema sowie weiteren Bereichen der Suchttherapie werden neben einem vielfältigen Angebot suchttherapeutischer Konzeptionen und Themen grundlegende und aktuelle Therapieformen vorgestellt, diskutiert und intensiv erarbeitet: Entgiftungsbehandlung, Pharmakotherapie, Nikotinabhängigkeit, Kokain und Crack, Komorbidität, Suchtprävention, kunsttherapeutische Praxis sind Bestandteile des Seminarkongresses ebenso wie Familientherapie, Psychoedukation und Motivationsförderung.

\section{Veranstalter}

Zentrum für Interdisziplinäre Suchtforschung der Universität Hamburg (ZIS), Institut für interdisziplinäre Sucht- und Drogenforschung (ISD, Hamburg), Klinik und Poliklinik für Psychiatrie und Psychotherapie, Universitätsklinikum Hamburg-Eppendorf u.a.

\section{Weitere Informationen und das Programmheft erhalten Sie über das}

Kongressbüro „Suchttherapietage“, Zentrum für Interdisziplinäre Suchtforschung der Universität Hamburg, Klinik für Psychiatrie, UKE, Martinistr. 52, D-20246 Hamburg. Fax: +49-40-42803-5121 E-mail: kontakt@suchttherapietage.de 